\title{
Evaluation of the Calculable High Frequency AC-DC Standard
}

\author{
Cees J. van Mullem, Willemien J. G. D. Janssen, and Jan P. M. de Vreede
}

\begin{abstract}
A calculable, high-frequency (HF) ac-dc transfer standard has been developed at NMi Van Swinden Laboratorium (VSL). An improved model which contains a more detailed description of the thermal element with the end disc, connecting leads, and the housing is presented together with the results of the ac-dc transfer measurements. The major result is a calculated shift of about $3400 \mu \mathrm{V} / \mathrm{V}$ in the ac-de difference at $100 \mathrm{MHz}$ of the $4 \mathrm{~V}$ HF ac-de standard. Based on this model, a $2 \mathrm{~V}$ standard is under development. The first results of this standard confirm the new modeling.
\end{abstract}

\section{INTRODUCTION}

$\mathbf{T}$ HE DEVELOPMENT and realization of an ac-dc transfer standard for the frequency range from $200 \mathrm{kHz}-100 \mathrm{MHz}$ with relatively low uncertainties has been carried out at NMi Van Swinden Laboratorium (VSL). The first results up to $30 \mathrm{MHz}$ have been published by Nomair and Harmans at CPEM' 88 [1], [2]. These investigations led to a $4 \mathrm{~V}$ calculable ac-dc standard with an extension in the frequency range up to $100 \mathrm{MHz}$. The existing model of the standard [1], [2] has been extended to $100 \mathrm{MHz}$ without any model changes. Four devices are used as the VSL primary reference group. Furthermore, VSL is producing this standard, and about 40 units have been sold to several national standards institutes.

However, an intercomparison with other institutes [3] has indicated potential discrepancies for the ac-dc transfer in the upper frequency range. Also, one of the fabricated standards (EUR-18) gives unexpectedly large deviations between the model and the measurements at high frequencies. This might be due to the fact that the constructed ac-dc standards are more or less identical, so systematic errors may have been left undetected. In the case of the EUR-18, the length of a connecting wire deviates from the average length of this wire in other standards we have made.

These assumed discrepancies between model and measurements was one of the reasons to develop a more detailed model of the standard [4]. The results are presented together with the uncertainty calculation of the model parameters of the standard. Also, the first results of the development of a $2 \mathrm{~V}$ version of the standard is given which are used to support the new modeling.

\section{Calculable HF AC-DC Standard}

The layout of the calculable HF ac-dc standard is given in Fig. 1. It consists of a range resistor (a thin $20 \mu \mathrm{m}$ diameter

Manuscript received June 20, 1996; revised October 1, 1996.

The authors are with the NMi Van Swinden Laboratorium (VSL), 2600 AR Delft, The Netherlands.

Publisher Item Identifier S 0018-9456(97)01645-8.

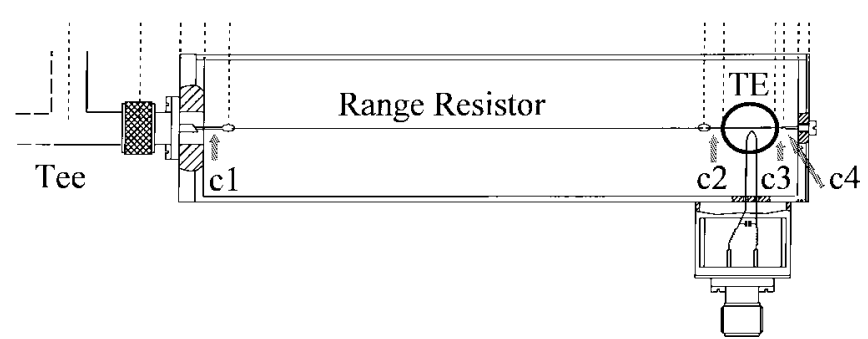

Fig. 1. The VSL calculable HF ac-dc standard. The dashed lines indicate the different sections which are used in the new modeling of the device including half of the Tee-connector and input $\mathrm{N}$-connector.

Isaohm wire) and a thermal element (TE), type UHF [Best Technology, a quaternay alloy (Evaohm) heater], which are mounted in a cylindrical brass housing with a diameter of $50 \mathrm{~mm}$. It is equipped with an N-type input connector. Several wires are applied to connect these different elements (see also Fig. 1):

c1 A copper wire between the input connector and the range resistor.

c2 A Pt-Ir feedthrough wire between the range resistor and the heater of the TE.

c3 A Pt-Ir feedthrough wire between the heater of the TE and copper wire 44 .

c4 A copper wire between the feedthrough wire of the TE and the end disc.

Thin solder points are used to connect the different parts. The connecting wires are of importance, in the new model, since they represent a considerable part of the total calculated ac-dc transfer difference.

\section{Modeling OF THE AC-DC STANDARD}

The original model, developed by Nomair and Harmans, is based on transimpedance theory. The model is built up by using four major blocks, two describing the input connector (distinction between air or teflon dielectric isolation), one describing the resistive wire and the last describing the thermal element as load for this wire. The TE is modeled as a lumped element.

This model is limited for several reasons. First, the influence of the connecting (copper) wires and the feedthrough wires of the thermal element have been underestimated. Second, because of software limitations, approximations for sinh and cosh terms are used in the calculations. Finally, due to the way the system is described, it is not easy to investigate the separate influence of the different parts of the model. 


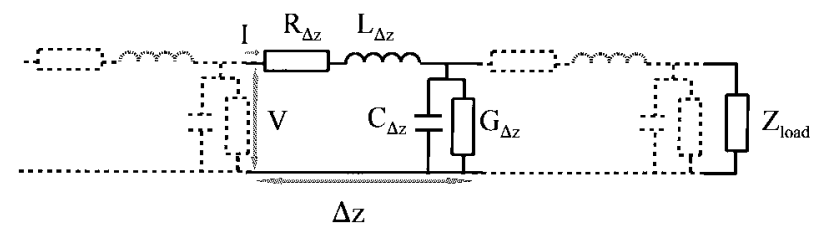

Fig. 2. Electrical scheme of a basic section of a coaxial transmission line.

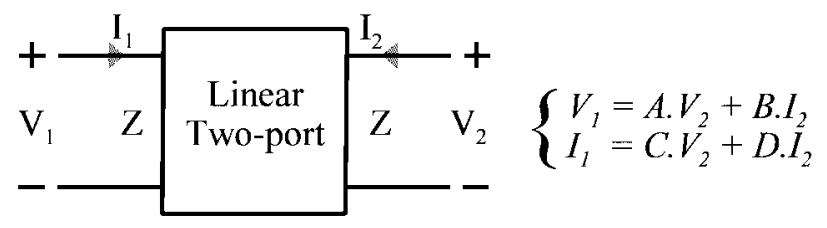

Fig. 3. $A B C D$-representation of a basic section.

The new model [4] assumes that the standard is a coaxial transmission line with losses, consisting of an inner and an outer conductor, loaded by the impedance $Z_{\text {load }}$. This coaxial line is divided into infinitesimal sections of length $\Delta z$. The electrical characteristics of such a basic section is represented by a resistance and an inductance in series, and a capacitance and a conductance in parallel, see Fig. 2. The definition of a section is given by the geometric properties of the standard. There are 10 sections defined, see also Fig. 1. Knowing the mechanical and the electrical properties of a section, the corresponding propagation factor $(\gamma)$ and the characteristic impedance $Z_{o}$ can be calculated (the conductance is neglected). In this calculation, the skin effect has been taken into account for the resistance and the inductance. The TE is still modeled as a lumped element.

The new model of the total system is based upon $A B C D$ parameters which can be converted into scattering parameters [5]. The main advantages of the $A B C D$-parameters are the possibility to calculate the influence of each separate section and its accessibility. Also by using these parameters, the separate sections can be connected even if they are not loaded by the characteristic impedance. Each section is described as a linear two-port with the given input/output relation (see Fig. 3). The $A B C D$-parameters of a transmission line section with losses is given by [5]:

$$
\begin{aligned}
& A=\cosh (\gamma \cdot l) \\
& B=Z_{o} \cdot \sinh (\gamma \cdot l) \\
& C=\frac{\sinh (\gamma \cdot l)}{Z_{O}} \\
& D=\cosh (\gamma \cdot l)
\end{aligned}
$$

with $l$ the length of the corresponding section.

The total $A B C D$ matrix of the complete system is calculated by matrix multiplication. The system transimpedance $\left(Z_{\mathrm{tr}, \text { system }}\right)$ is determined from this total ABCD matrix with the thermal element as load impedance, according to:

$$
Z_{\mathrm{tr}, \text { system }}=A_{\text {total }} \cdot Z_{\text {load }}+B_{\text {total }} .
$$

Furthermore, the ac power dissipation in the heater of the TE is calculated. This is referred to the dc power dissipation to

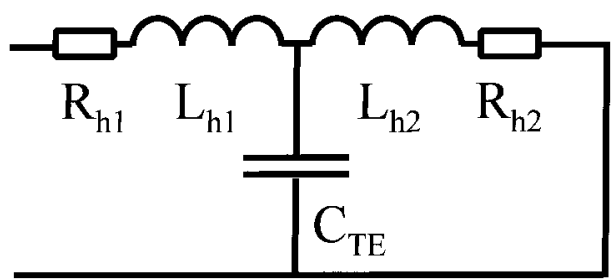

(a)

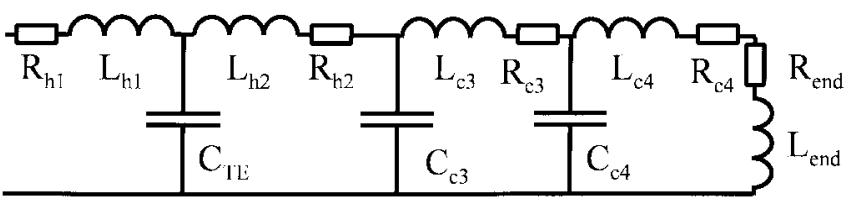

(b)

Fig. 4. Modeling of the thermal element and the connection between heater and end disc (a) model 1 and (b) model 2.

determine the ac-dc difference $(\delta)$ of the standard [2]:

$$
\begin{aligned}
\delta & =\left.\frac{V_{\mathrm{ac}}-V_{\mathrm{dc}}}{V_{\mathrm{dc}}}\right|_{P_{\mathrm{ac}}=P_{\mathrm{dc}}} \\
& =\left|Z_{\text {tr }, \text { system }}\right| \cdot \sqrt{\frac{R_{h, \mathrm{dc}}}{R_{h, \mathrm{ac}}}} \cdot \frac{1}{R_{\mathrm{dc}} \cdot Y}-1
\end{aligned}
$$

with $R_{h, \mathrm{dc}}$ and $R_{h, \text { ac }}$ the dc and ac heater resistance, respectively, $R_{\mathrm{dc}}$ the system dc-resistance and $Y$ representing the ac current efficiency of the heater which is mainly determined by the capacitive loss of the heater.

The main result is an improved model which has a significant shift of the calculated ac-dc transfer difference especially for the higher frequency region. This shift is a result of:

1) Modeling of the capacitive loading due to the connecting wires, all capacitive loads are taken into account.

2) Detailed modeling of the TE in combination with the end disc. Model 1 describes only the influence of the heater, neglecting the connecting wires, see Fig. 4(a). It means, only the heater resistance $\left(R_{h 1}\right.$ and $\left.R_{h 2}\right)$, the heater inductance $\left(L_{h 1}\right.$ and $\left.L_{h 2}\right)$ and the heater capacitance $\left(C_{\mathrm{TE}}\right)$ are modeled. On the other hand, model 2 also describes the influences of the connection between the TE and the end disc as well, see Fig. 4(b). The model is extended with representative electrical elements for the connecting wires $\mathrm{c} 3\left(R_{\mathrm{C} 3}, L_{\mathrm{c} 3}\right.$, and $\left.C_{\mathrm{c} 3}\right)$ and $\mathrm{c} 4\left(R_{\mathrm{c} 4}\right.$, $L_{\mathrm{c} 4}$, and $C_{\mathrm{c} 4}$ ).

3) Modeling of the end disc by a resistance $\left(R_{e n d}\right)$ and an inductance $\left(L_{e n d}\right)$ instead of a short-circuit as done in model 1 [Fig. 4(a)].

The second model still uses the mechanical dimensions and the measured dc-electrical parameters as input parameters. Table I presents the results of the calculation of a standard using the two models. It clearly shows the large differences between the two models, especially at high frequencies. The calculated difference at $100 \mathrm{MHz}$, around $3400 \mu \mathrm{V} / \mathrm{V}$, is of the same order as was found during the intercomparison of thermal converters at high frequencies [3]. The connecting copper wire at the input (c1) and the feedthrough lead between the range 
TABLE I

Modeled AC-DC DifFerence of THE CALCUlable AC-DC TRansfer Standard (EUR-36)

\begin{tabular}{l|c|c|c|c|c}
\hline \hline Frequency $(\mathrm{MHz})$ & 1 & 10 & 30 & 50 & 100 \\
\hline o_model 1 $(\mu \mathrm{V} / \mathrm{V})$ & 6 & 42 & 37 & -136 & -2389 \\
\hline$\delta$ model $2(\mu \mathrm{V} / \mathrm{V})$ & 6 & 7 & -281 & -1007 & -5710 \\
\hline \hline
\end{tabular}

TABLE II

Uncertainty ( $2 \sigma$ ) Calculation of the Model Parameters (Model 2)

\begin{tabular}{l|c|c|c|c|c}
\hline \hline Frequency $(\mathrm{MHz})$ & 1 & 10 & 30 & 50 & 100 \\
\hline Uncertainty $(\mu \mathrm{V} / \mathrm{V})$ & $<1$ & 5 & 40 & 125 & 500 \\
\hline \hline
\end{tabular}

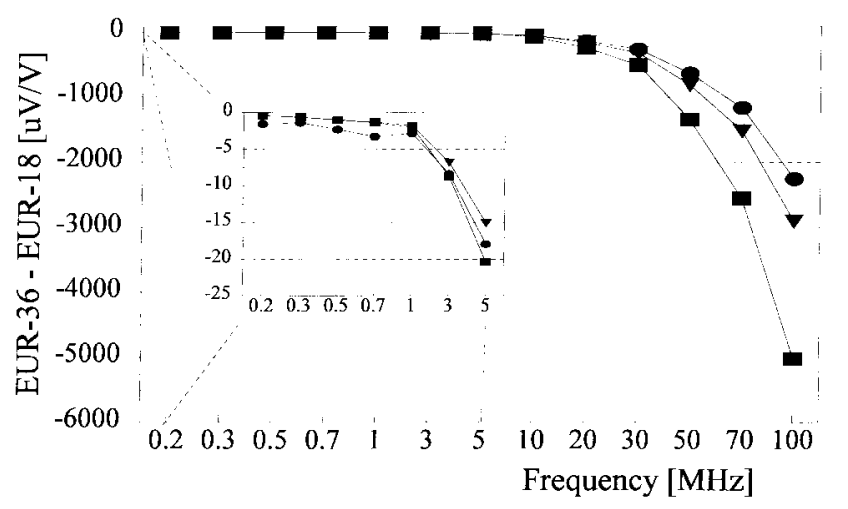

- Model 1 - Model 2 Measurements

Fig. 5. Calculated and measured ac-dc difference between the two standards $(4 \mathrm{~V})$ from $200 \mathrm{kHz}-100 \mathrm{MHz}$. The inserted figure gives a detailed view from $200 \mathrm{kHz}-5 \mathrm{MHz}$.

resistor and TE (c2) are mainly responsible for the shift in the ac-dc transfer difference, about $25 \%$ and $60 \%$, respectively.

\section{UnCERTAinTy CALCULATION OF THE MODEl PARAMETERS USING MODEL 2}

The uncertainty calculation of the model parameters has been performed for both models. It includes the uncertainties of the mechanical dimensions and the dc-electrical parameters, and the estimation of the conductivity of the materials used and the capacitance of TE. The results of model 2 are presented in Table II. The total uncertainty is mainly determined by the thickness of the front disc, the length of the connecting wire to the range resistor $(\mathrm{c} 1)$, the input feedthrough (c2), the diameter of the cylinder and finally the resistance of the range resistor and the heater, respectively. Of course, the total measurement uncertainty will include the accuracy of the model itself and the uncertainty of the connectors and the measurement set-up [6], [7].

\section{MEASUREMENTS}

The measurement setup used is the (automated) VSL ac-dc measurement system using a comparator principle to obtain immunity to variations in the ac or dc input voltage [8].

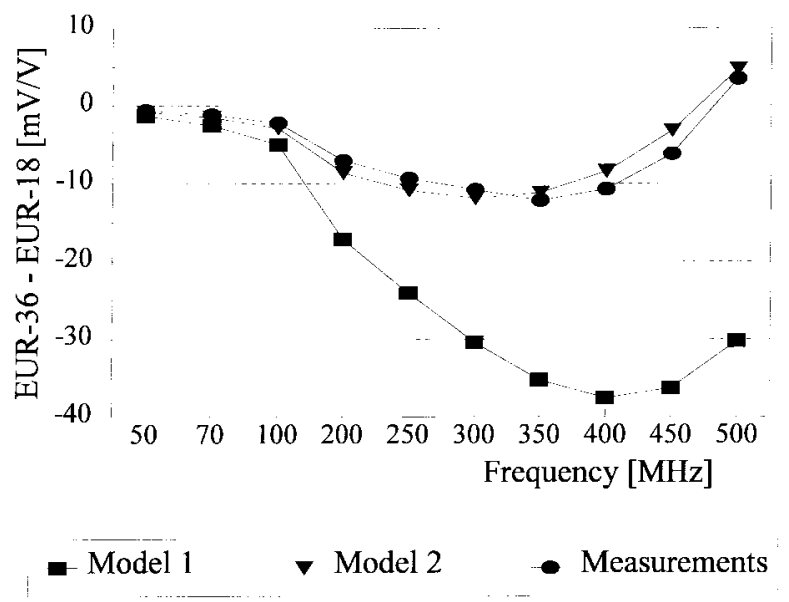

Fig. 6. Calculated and measured ac-dc difference between the two standards (4 V) from $(50-500) \mathrm{MHz}$.

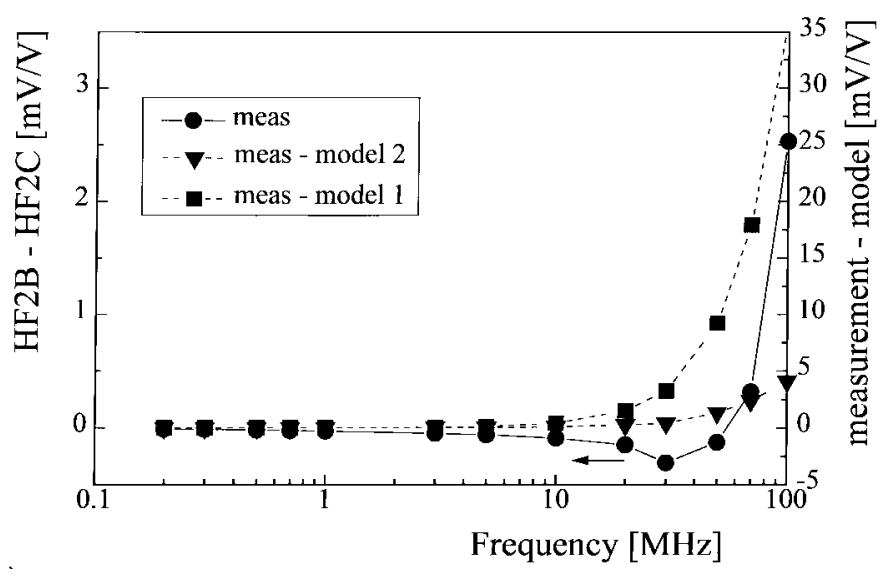

Fig. 7. Calculated and measured ac-de difference between the two standards (2 V) from $200 \mathrm{kHz}-100 \mathrm{MHz}$.

The frequency range is extended to $500 \mathrm{MHz}$ because the main differences should be observed at $30 \mathrm{MHz}$ and higher frequencies.

First, the standard (EUR-18) with the divergent behavior at high frequencies is measured against a "normal" standard (EUR-36). In Figs. 5 and 6 the results are given for two frequency ranges (up to $100 \mathrm{MHz}$ and from (50-500) $\mathrm{MHz}$ ). The measurements and the calculated results of model 2 show a good agreement. The results predicted by model 1 have a much larger deviation from the measurements. This is caused by the insufficient detailed modeling of the connecting wires; the EUR-18 standard has a relatively long connecting wire between the input connector and the range resistor and, consequently, a shorter length of this resistive wire.

Altogether, more than 50 devices produced by VSL have been measured against one of our reference standards. The measurements show a good agreement for both models in the case of two more or less identical devices. The average deviation between measurement and model is about $800 \mu \mathrm{V} / \mathrm{V}$ at $100 \mathrm{MHz}$ with a standard deviation of $260 \mu \mathrm{V} / \mathrm{V}$. However, model 2 has an absolute calculated difference of $3400 \mu \mathrm{V} / \mathrm{V}$ $(100 \mathrm{MHz})$ with model 1 . Therefore, new standards (which have nonidentical mechanical construction) are being devel- 
oped for a more accurate investigation of the systematic errors in the modeling.

\section{DEVELOPMENT OF A 2 V STANDARD}

The $2 \mathrm{~V}$ standard is based on the present design of the $4 \mathrm{~V}$ standard but with a smaller value for the range resistor. Three devices have been made having nonidentical mechanical dimensions. These types are:

HF2A Using a thicker resistive wire.

HF2B Using a shorter resistive wire by shortening the housing of the standard.

HF2C Using a shorter resistive wire by increasing the length of the copper connecting wire between input and range resistor.

The measurement results of a $2 \mathrm{~V}$ standard against a $4 \mathrm{~V}$ standard deviate more from the calculated value up to a difference of $5 \mathrm{mV} / \mathrm{V}$ at $100 \mathrm{MHz}$. This is due to the relatively large ac-dc transfer differences between the (2 and 4) $\mathrm{V}$ standard, $60 \mathrm{mV} / \mathrm{V}$.

The measurement of the standard HF2B against the HF2C indicates the improved modeling of the standard. In Fig. 7 the measurement and the difference between measurement and modeling of the two standards are given. The solid line presents the measurement, the dashed lines indicate the difference between the measurement and modeling, as well as for model 1 as for model 2 . It is clearly shown that model 1 does not predict the correct frequency behavior, there is more than $35 \mathrm{mV} / \mathrm{V}$ difference at $100 \mathrm{MHz}$ for model 1 compared with $4 \mathrm{mV} / \mathrm{V}$ for model 2 . It is caused by the fact that model 1 does not take into account the effect of the capacitive coupling of the copper connecting wire, which is relatively long for standard $\mathrm{HF} 2 \mathrm{C} ; 100 \mathrm{~mm}$ compared to $12 \mathrm{~mm}$ for standard HF2B.

The further development of the $2 \mathrm{~V}$ standard will concentrate on the optimization of the mechanical dimensions to decrease the ac-dc error at the higher frequencies in such a way that it becomes comparable to the $4 \mathrm{~V}$ standard to be able to perform accurate measurements for high frequencies and, subsequently, further refinements of our model. After this development, comparisons with other type of rf standards will be made to obtain more refinements to the model.

\section{CONCLUSIONS}

The evaluation of the VSL calculable HF ac-dc standard (4 V) has led to an improved model, which mainly gives a better description of the high frequency behavior of the devices. This is confirmed by a better agreement with our measurements and those of an earlier intercomparison. It clearly shows that the old model contains a systematic error which is about $3400 \mu \mathrm{V} / \mathrm{V}$ at $100 \mathrm{MHz}$. Good agreement between the measurements has been made on the 50 standards so far produced. The $2 \mathrm{~V}$ standards developed have verified the improvements made to the model of the HF ac-dc standard.

\section{REFERENCES}

[1] M. Nomair and C. J. Harmans, "High accuracy calculable ad-dc transfer standards for the LF-30 MHz frequency range," IEEE Trans. Instrum. Meas., vol. 38, pp. 342-345, 1989.

[2] _ "A high accuracy calculable ac-dc transfer standard," Delft, The Netherlands, VSL Rep. ES-88-04, 1989.

[3] J. R. Kinard, Z. Zhen, D.-X. Huang, G. Rebuldela, D. Janik, and J. de Vreede, "Intercomparison of thermal converters at NIM, NIST, PTB, SIRI, and VSL from 10 to $100 \mathrm{MHz}$," IEEE Trans. Instrum. Meas., vol. 42, pp. 618-621, 1993.

[4] W. J. G. D. Janssen, "Modeling of high frequency voltage converters," Delft, The Netherlands, VSL Int. Rep., 1994, in Dutch.

[5] P. A. Rizzi, Microwave Engineering, Passive Circuits. Englewood Cliffs, NJ: Prentice-Hall, 1988.

[6] J. R. Kinard and T.-X. Cai, "Determination of ac-dc difference in the 0.1-100 MHz frequency range," IEEE Trans. Instrum. Meas., vol. 38, pp. $360-367,1989$

[7] D.-X. Huang, M.-L. Chen, and S.-Z. He, "RF-DC differences of coaxial thermal standards," IEEE Trans. Instrum. Meas., vol. 39, pp. 313-317, 1990.

[8] C. Harmans, J. J. Schmitt, and R. Kaarls, "A high precision automated system for ac-dc transfer using thermal converters," in Dig. CPEM'82, 1982, pp. D5-D7.

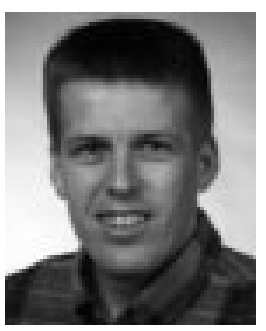

Cees J. van Mullem was born in Gouda, The Netherlands, in 1964. He received the M.Sc. and Ph.D. degrees in electrical engineering from the University of Twente, Enschede, The Netherlands, in 1989 and 1993, respectively.

In 1994, he joined the Department of Electricity and Magnetism, NMi Van Swinden Laboratorium (the Dutch national standards laboratory), Delft, The Netherlands. His main interests are the development and maintenance of ac-dc transfer standards, especially in the frequency range from $100 \mathrm{kHz}-100$ $\mathrm{MHz}$, and the calibration of ac-dc transfer and ac measuring instruments.

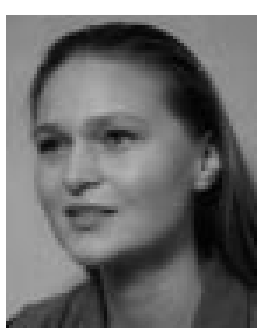

Willemien J. G. D. Janssen was born in Wamel, The Netherlands, in 1970. She received the B.Sc. degree in electrical engineering in 1994. During her degree work at the NMi Van Swinden Laboratorium, Delft, The Netherlands, she developed a new model for high-frequency ac-dc voltage converters.

After her graduation, she joined the Department of Electricity and Magnetism, National Standards Laboratory, Delft. Her main interests are the development and maintenance of high-frequency and electromagnetic standards and calibration facilities.

Jan P. M. de Vreede was born in Pijnacker, The Netherlands, in 1945. He received the M.S. and Ph.D. degrees in physics from the Rijksuniversiteit Utrecht, Utrecht, The Netherlands, in 1970 and 1977, respectively.

His postgraduate work at the University of Utrecht, from 1977 to 1983, involved high-precision microwave spectroscopy using FM-detection techniques. In 1983, he joined the Electrical Standards Department, Van Swinden Laboratorium (the Dutch national standards laboratory), Delft, The Netherlands. Since 1988, he has been Deputy Manager of this department. His main interests are the development and maintenance of high-frequency and fiberoptical standards and calibration facilities; hence he has been involved in several international comparisons in this field. 